\title{
Highly Efficient State-Selective Submicrosecond Photoionization Detection of Single Atoms
}

\author{
F. Henkel, ${ }^{1, \text { * }}$ M. Krug, ${ }^{1}$ J. Hofmann, ${ }^{1}$ W. Rosenfeld, ${ }^{1}$ M. Weber,${ }^{1}$ and H. Weinfurter ${ }^{1,2}$ \\ ${ }^{1}$ Fakultät für Physik, Ludwig-Maximilians-Universität München, D-80799 München, Germany \\ ${ }^{2}$ Max-Planck-Institut für Quantenoptik, D-85748 Garching, Germany \\ (Dated: May 28, 2021)
}

\begin{abstract}
We experimentally demonstrate a detection scheme suitable for state analysis of single optically trapped atoms in less than $1 \mu \mathrm{s}$ with an overall detection efficiency $\eta$ exceeding $98 \%$. The method is based on hyperfine-state-selective photoionization and subsequent registration of the correlated photoion-electron pairs by coincidence counting via two opposing channel electron multipliers. The scheme enables the calibration of absolute detection efficiencies and might be a key ingredient for future quantum information applications or precision spectroscopy of ultracold atoms.
\end{abstract}

PACS numbers: 03.67.-a, 32.80.Fb, 37.10.Jk, $42.50 \mathrm{Dv}$

One crucial requirement for quantum computation, quantum communication, and quantum metrology is the highly efficient measurement of qubit states. For atomic qubits, the most frequently used fluorescence method allows measuring with a detection efficiency of almost unity, however, at the cost of comparably long detection times $(>100 \mu \mathrm{s})$. In order to reduce the measurement duration for such systems one can either increase the numerical aperture of the collection optics [1] or, alternatively, enhance fluorescence by means of optical cavities [2].

Yet, if one intends to apply these approaches for state analysis of many atoms, e.g. for one-way quantum computation in optical lattices [3], difficulties arise. Even with fluorescence collection pushed to the limit [1] the detection times can hardly be reduced below $10 \mu \mathrm{s}$ per atom. Alternatively, optical cavities allow detection times below $1 \mu \mathrm{s}$ [4]. However, due to the typically small mode volume and reduced optical access, scalability to a large number of atoms may be challenging with current state of the art technologies. Therefore, in order to obtain both scalability and speed, a completely different approach is required.

In this Letter, we experimentally demonstrate how hyperfine-state-selective photoionization and subsequent detection of the generated photoion-electron pairs enable fast and efficient state analysis. On the example of single ${ }^{87} \mathrm{Rb}$-atoms, we reach a readout fidelity of $\mathcal{F}=99.2 \%$, an overall detection time below $1 \mu \mathrm{s}$, and an overall detection efficiency $\eta$ exceeding $98 \%$.

In a first experiment, efficiency, speed and hyperfinestate selectivity of the photoionization procedure are studied [5]. For this purpose, a single ${ }^{87} \mathrm{Rb}$ atom is loaded into a far off resonance optical dipole trap in an UHV glass cell setup, similar to [6] [Fig.[1(a)]. State selectivity is achieved by a two-step, two-color photoionization scheme using the $5^{2} P_{3 / 2}, F^{\prime}=3$ level as resonant, intermediate state [Fig.1 b) $, \lambda_{12}=780 \mathrm{~nm}, \lambda_{2 \mathrm{i}}=473 \mathrm{~nm}$ ]. For that purpose two perpendicular, focused laser beams $\left(w_{12}=\right.$ $\left.54 \mu \mathrm{m}, P_{12}=6 \mu \mathrm{W} ; w_{2 \mathrm{i}}=1.13 \mu \mathrm{m}, P_{2 \mathrm{i}}=32.8 \mathrm{~mW}\right)$ are overlapped with the optical dipole trap into one common focus [Fig.11a)]. The polarizations of both laser fields (a)

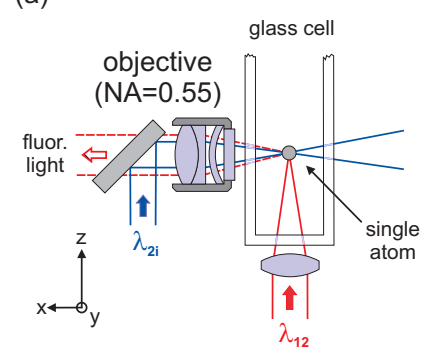

(c)

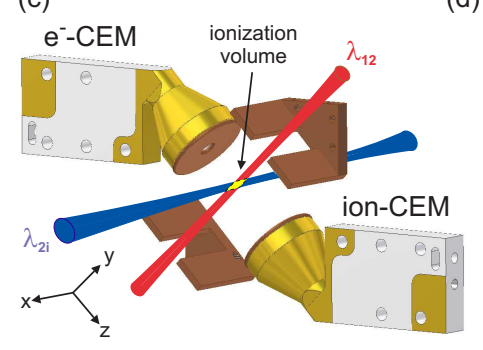

(d)
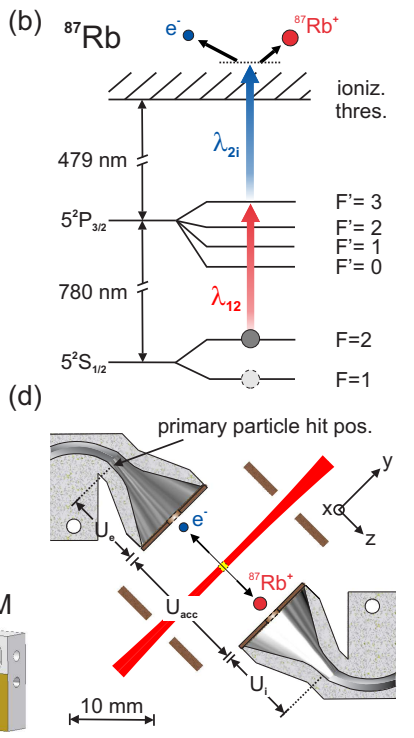

Figure 1. (color online). (a) Setup for single atom ionization: Single atoms are trapped in an optical dipole trap, prepared into selected hyperfine states and subsequently ionized. (b) Photoionization level scheme: Single ion-e ${ }^{-}$pairs are generated by hyperfine-state-selective, resonant two-step, two-color photoionization. (c) Joint channel electron multiplier (CEM) detector: two opposing CEMs and compensation electrodes against stray fields are built into a glass cell identical to (a). (d) Detector geometry in section view (laser beam waist not to scale).

are linear and parallel to the y-axis. During measurements no electric field is applied and only small magnetic fields $(<50 \mathrm{mG})$ are present.

To evaluate the photoionization process, we investigate the ionization probability of the trapped atom for different excitation pulse lengths (Fig. (2). By optical pumping, the atom is initially prepared either in the $5^{2} S_{1 / 2}, F=1$ or $F=2$ hyperfine ground state, respectively [Fig.11b)]. Then, pulses of the excitation and ionization light are simultaneously applied. While the ionization pulse length is fixed to $2.12 \mu \mathrm{s}$ for all measurements, the excitation pulse length $t_{\mathrm{p}}$ is varied between $36 \mathrm{~ns}$ and $2.0 \mu \mathrm{s}$ (Fig.2, inset). 


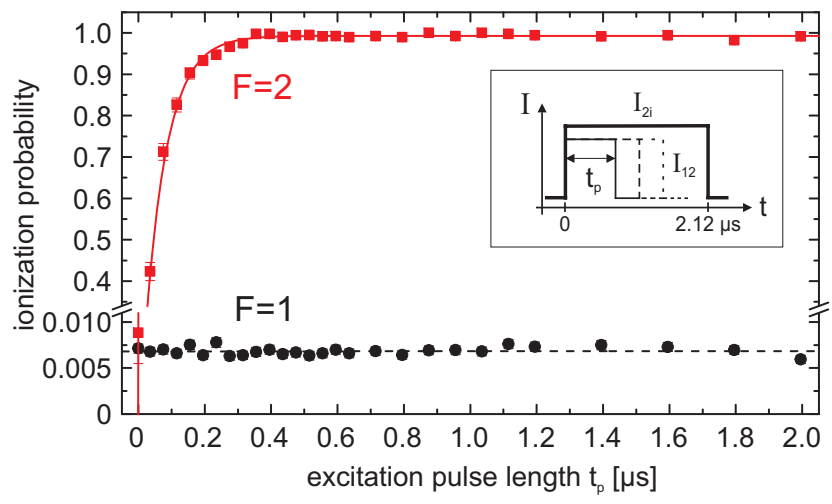

Figure 2. (color online). Hyperfine-state-selective, single atom ionization probability for different laser pulse lengths. Atoms in the trap are initially prepared either in the $5^{2} S_{1 / 2} F=2(\square)$ or the $F=1$ (०) hyperfine state, respectively. Only atoms in $F=2$ driven by both laser fields $\left(\lambda_{12}, \lambda_{2 \mathrm{i}}\right)$ are ionized. Inset: Scheme showing the timing of the excitation and ionization pulses.

The excitation pulse operates at a multiple of the saturation intensity of the cycling transition, yielding the $5^{2} P_{3 / 2}$ state population approaching one-half after a few lifetimes of the excited state $\left(\tau_{\text {exc }}=26.2 \mathrm{~ns}\right)$.

Figure 2 shows the ionization probability after application of a single excitation-ionization sequence. As the ionization process removes the atom out of the trap, the ionization probability is derived from atom loss, analyzed by subsequent fluorescence detection. For atoms initially prepared in $F=2$, the dynamics of the resonant two-step, two-color photoionization is described by a rate equation model [7], valid for $t_{p}$ longer than the lifetime of the intermediate $5^{2} P_{3 / 2}$ level. The ionization probability is

$$
p_{\text {ion, } \mathrm{F}=2}\left(t_{p}\right)=p_{\infty}\left(1-\exp \left[-\rho_{\mathrm{ee}} \sigma_{2 \mathrm{i}} \Phi_{2 \mathrm{i}} t_{p}\right]\right),
$$

with $p_{\infty}$ being the probability to ionize the atom for $t_{p} \rightarrow$ $\infty, \rho_{\mathrm{ee}} \approx \frac{1}{2}$ the steady-state population of the $5^{2} P_{3 / 2}$ level, $\sigma_{2 \mathrm{i}}$ the ionization cross section [8], and $\Phi_{2 \mathrm{i}}$ the photonic flux of the ionizing laser. For the evaluation of $p_{\text {ion, } \mathrm{F}=2}$, the parameters $\rho_{\mathrm{ee}}, \sigma_{2 \mathrm{i}}$ and $\Phi_{2 \mathrm{i}}$ are combined into a characteristic $\frac{1}{e}$-ionization time $\tau=\left(\rho_{\mathrm{ee}} \sigma_{2 \mathrm{i}} \Phi_{2 \mathrm{i}}\right)^{-1}$. To determine $\tau$, a least-square fit according to (1) is applied for pulse lengths $t_{p}$ up to $475 \mathrm{~ns}$ (red line, Fig.2). With $p_{\infty}=0.993 \pm 0.001$ deduced by averaging the measured ionization probabilities for $t_{p}>475 \mathrm{~ns}$, we obtain $\tau=64.4 \pm 2.8 \mathrm{~ns}$. Thus, after an ionization time $t_{\text {ion }} \equiv$ $6 \tau=386 \mathrm{~ns}$ ( $F=2$, Fig.2), an ionization probability of $p_{\text {ion, } \mathrm{F}=2}\left(t_{\text {ion }}\right)=0.9905 \pm 0.0010$ is achieved. Other loss mechanisms, as, e.g., possible single-color, multiplephoton ionization processes or heating are considered. For that purpose each of the beams is switched on separately, showing negligible ionization probabilities. In order to demonstrate the hyperfine-state selectivity of the ionization scheme, we compare this result with measurements for the atom initially prepared in the $5^{2} S_{1 / 2}, F=1$ state. Here, we observe a probability $p_{\text {ion,F=1 }}$ of $0.0068 \pm 0.0001$ for all excitation pulse lengths $t_{\mathrm{p}}(F=1$, Fig.(2).

From these measurements, a readout fidelity $\mathcal{F}=\frac{1}{2}\left[p_{\text {ion } \mathrm{F}=2}+\left(1-p_{\text {ion, } \mathrm{F}=1}\right)\right]$, defined as the average probability to correctly identify the hyperfine state, can be deduced, resulting in a value of $\mathcal{F}\left(t_{\text {ion }}\right)=99.19 \pm 0.05 \%$ [9]. Note, this value also includes preparation errors, atom loss out of the trap, and errors of the fluorescence detection and since the observed $p_{\text {ion, } \mathrm{F}=1}$ is indistinguishable from measurements without any ionizing light the actual readout fidelity of the ionization process is even closer to unity.

In a second experiment, the efficiency and time required for detecting the generated photoion-electron pairs, as well as the sensitive volume of the detection system are investigated. To detect both ionization fragments, this system consists of two channel electron multipliers (CEMs, [10]) whose cone entrances are separated by $d=15.7 \mathrm{~mm}$. To protect the CEM cones against stray light and to tailor the electric fields inside the cones, the entrances are covered by copper plates with an open aperture of $2 \mathrm{~mm}$. Additional electrodes next to the CEMs compensate for electric stray fields [Fig.11(c)]. This system is mounted in a separate UHV glass cell setup, where in between both detectors at $z=d / 2,{ }^{87} \mathrm{Rb}$-atoms from the thermal background vapor are photoionized within the overlap of two mutually perpendicular laser beams. The overlap region can be moved in all three dimensions in order to investigate the spatial dependence of the detection efficiency (the sensitive volume). Uniform ionization conditions over the scan region are provided by only weakly focusing the beams $\left(w_{12}=26 \mu \mathrm{m}\right.$, $\left.P_{12}=155 \mu \mathrm{W} ; w_{2 \mathrm{i}}=43 \mu \mathrm{m}, P_{2 \mathrm{i}}=164 \mathrm{~mW}\right)$. To separate the oppositely charged photoionization fragments into their corresponding CEM, the CEMs are held at different electric potentials defining the total acceleration voltage $U_{a c c}$ [Fig.11(d)] which in turn determines the kinetic energy $E_{\text {kin }}$ of the fragments at the cone entrance [11]. The particular arrangement of the CEMs and the compensation electrodes ensures that no additional electron/ion optics is required, thus allowing a large solid angle for optical access.

In order to determine the duration $t_{\mathrm{det}}$ of the detection process, the time from the ionization event to the detection of the macroscopic pulses at the anodes of the CEMs is investigated [12]. It is composed of the respective flight times $\left(t_{e}, t_{i}\right)$ of the two ionization fragments until the primary particle hit in the corresponding detector [Fig.11(d)] and the transit time of the electron avalanche inside the CEM channels. In the experiment only the time-of-flight difference $\Delta t=t_{i}-t_{e}$ is accessible [Fig.3 (a)]. It is deduced from a Gaussian fit of the detection time differences [Fig.3(a), inset]. The temporal spread of the correlation peak [indicated by the error bars in Fig.3 (a)] remains narrow for a wide range of acceleration voltages $(1.6-3.8 \mathrm{kV})$ with FWHM $\leq 8.5 \mathrm{~ns}$. The measured time-of-flight differences can be modeled assuming acceleration of the ion or electron in a homogeneous field 

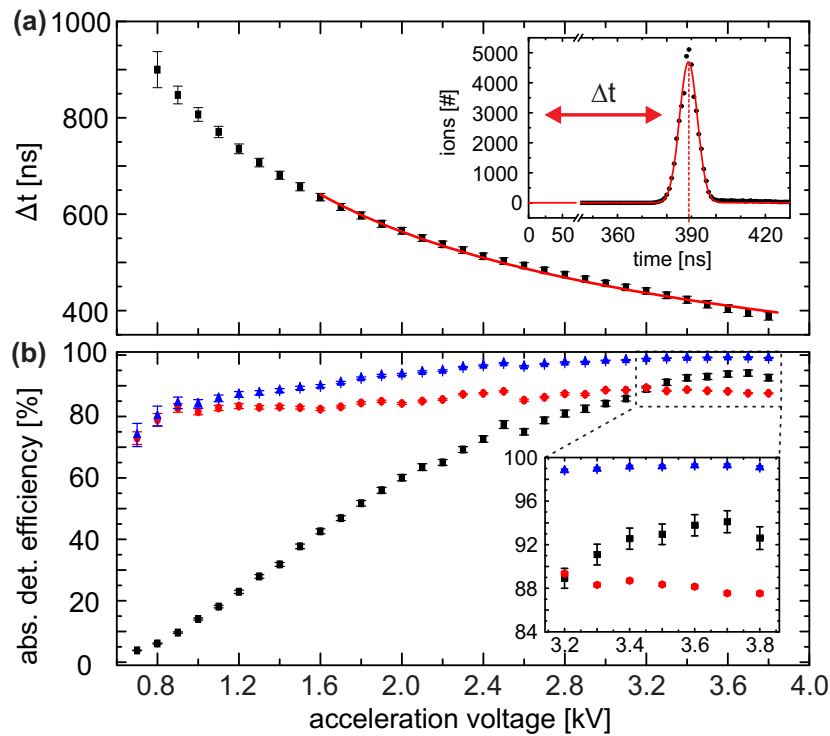

Figure 3. (color online). (a) Time-of-flight difference $\Delta t$ of generated ${ }^{87} \mathrm{Rb}$-ions to their corresponding photoelectrons for different acceleration voltages $U_{a c c}$. Inset: Sample histogram of time differences between ${ }^{87} \mathrm{Rb}$-ion and electron detections for $U_{a c c}=3.8 \mathrm{kV}$. (b) Absolute detection efficiency for ${ }^{87} \mathrm{Rb}$-ions ( $\square$, black), electrons ( $\circ$, red) for different acceleration voltages and the calculated, total detection efficiency $(\triangle$, blue). Inset: Zoom for acceleration voltages from 3.2 to $3.8 \mathrm{kV}$.

$E_{\text {acc }}$ up to the CEM entrance and further deceleration (acceleration) within the respective CEM. The model holds for $U_{a c c}$ above $1.6 \mathrm{kV}$ [red line, Fig 3 a)], below this value the actual field configurations between and inside the CEMs have to be taken into account in more detail. At $U_{a c c}=3.8 \mathrm{kV}$, we observe a time-of-flight difference of $\Delta t=388.81 \pm 0.01 \mathrm{~ns}$. According to the model we obtain a photoelectron flight time of $t_{e}=0.95 \mathrm{~ns}$ for this acceleration voltage, which together with the CEM transit time of $26 \mathrm{~ns}$ [10] sums up to a detection time of $t_{\text {det }}=\Delta t+t_{e}+t_{\text {transit }}=415.8 \mathrm{~ns}$.

For many applications the crucial parameter is a high detection efficiency [13, 14], i.e. in our case the total efficiency of collecting the respective fragment into the CEM and converting it into an observable electron avalanche. In order to optimize the efficiency for our detection system, the positions of the primary particle hit inside the CEMs are designed to be under grazing incidence at the channel walls [Fig.1(d)] and not in the CEM cones [15, 16]. Absolute detection efficiencies can be determined by coincident detection of the oppositely charged ionization fragments with two particle detectors [17]. Such a calibration is in perfect conceptual correspondence to $4 \pi \beta \gamma$-coincidence counting [18] or absolute photodetector calibration via photon pairs [19]. Accordingly, the CEM ion and $\mathrm{e}^{-}$-detection efficiencies $\eta_{i}, \eta_{e}$ are given by

$$
\eta_{i}=\frac{N_{c}}{N_{e}^{\prime}}, \quad \eta_{e}=\frac{N_{c}}{N_{i}^{\prime}}
$$

where $N_{i}^{\prime}=N_{i}-N_{b i}$ and $N_{e}^{\prime}=N_{e}-N_{b e}$ are the background corrected ion and $\mathrm{e}^{-}$single counts and $N_{c}$ is the number of coincidences. The latter is obtained from the detection time difference histograms [Fig.3 (3), inset] with a coincidence time window starting $20 \mathrm{~ns}$ before the correlation peak and ending $80 \mathrm{~ns}$ after it. This choice results from the presence of a few late ion detections. Accidental coincidences can be neglected, as the fraction of accidental to true coincidences is smaller than $10^{-4}$. The background events $N_{b i}, N_{b e}$ are measured with the excitation laser turned off, leaving only the ionization laser switched on. Figure 3 (b) shows the absolute detection efficiencies for ions and electrons for different acceleration voltages $U_{a c c}$. For values up to $3.8 \mathrm{kV}$, the ion detection efficiency $\eta_{i}$ increases in qualitative agreement with previous studies for different ion species [16, 20]. In contrast to this, the measured electron detection efficiency $\eta_{e}$ remains almost constant. At $U_{a c c}=3.8 \mathrm{kV}$, for a measurement time of $60 \mathrm{~s}$ we observe $N_{i}=53762, N_{b i}=2235, N_{e}=196547$, $N_{b e}=147845$ and $N_{c}=45099$ (including ion and electron dark counts $N_{d i} \sim 2100$ and $N_{d e} \sim 15000$, respectively), resulting in an absolute CEM detection efficiency of $\eta_{i}=0.926 \pm 0.010$ and $\eta_{e}=0.875 \pm 0.002$ [21]. From these efficiencies, a total efficiency for the detection of a photoionized neutral atom can be determined. As it is sufficient to detect the photoion or the photoelectron, the total detection efficiency $\eta_{\text {det }}$ is

$$
\eta_{\mathrm{det}}=1-\left(1-\eta_{i}\right)\left(1-\eta_{e}\right)=\eta_{e}+\eta_{i}-\eta_{i} \eta_{e} .
$$

Using the above values at $3.8 \mathrm{kV}$, a total detection efficiency of $\eta_{\mathrm{det}}=0.991 \pm 0.002$ is derived.

Finally, we analyze the spatial dependence of the detection efficiency, i.e., the sensitive volume of the detection system. Figure 4 a) shows the spatial distribution of the efficiency determined by a 2D scan in the x-y plane at $d / 2$ in between both CEMs. Here, for both detectors, a roughly circular distribution is observed. A scan along the $\mathrm{x}$ direction through the center of the area is depicted in Fig. 4(b) for different CEM gain voltages at $U_{a c c}=3.8 \mathrm{kV}$. Uniform efficiencies over the full area [see $\mathrm{e}^{-}$-CEM, Fig. 4(b)] are achieved for high gain voltages [22], similar to previous experiments with electron and ion beams [16]. From these measurements we obtain a sensitive area with a diameter of $d_{\circ}=0.84 \mathrm{~mm}$ where $\eta_{\text {det }}$ exceeds $98.8 \%$. Corresponding measurements at different $\mathrm{z}$ positions display a similar spatial behavior, indicating that the sensitive volume has a longitudinal extension of at least $5 \mathrm{~mm}$. By moving the ionization region closer to the ion-CEM and adapting $U_{a c c}$, even shorter ion flight times can be achieved, retaining high detection efficiencies.

Combining all measurement results, an overall efficiency $\eta=p_{\text {ion, } \mathrm{F}=2} \eta_{\text {det }}$ for state-selective detection of a single, neutral atom stored in an optical dipole trap and its corresponding detection time $t_{\text {tot }}=t_{\text {ion }}+t_{\text {det }}$ can be estimated. At an acceleration voltage of $U_{a c c}=3.8 \mathrm{kV}$, an overall detection efficiency of $\eta=0.982 \pm 0.002$ with an overall detection time $t_{\text {tot }}=802 \pm 17 \mathrm{~ns}$ is determined. 
(a)

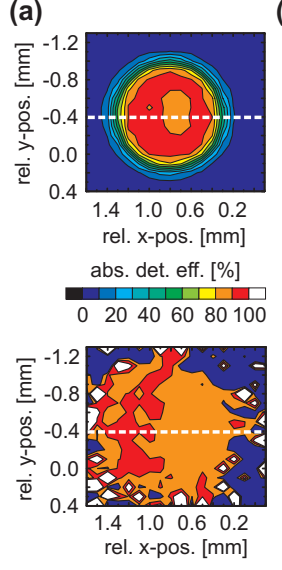

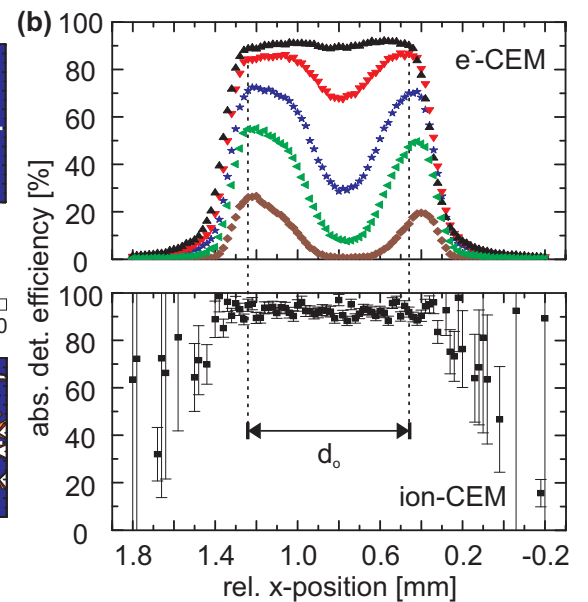

Figure 4. (color online). (a) 2D scans of the sensitive areas at a gain voltage of $2.8 \mathrm{kV}$ for the $\mathrm{e}^{-}$CEM (top) and ion CEM (bottom) for an acceleration voltage of $3.8 \mathrm{kV}$. Dashed lines indicate respective line scans in (b). (b) Line scans through the center at $y=-0.4 \mathrm{~mm}$. Electron detection efficiencies (top) at different gain voltages $(\diamond=2.4 \mathrm{kV}$ (brown); $\triangleleft=2.5 \mathrm{kV}$ (green); $\star=2.6 \mathrm{kV}$ (blue); $\nabla=2.8 \mathrm{kV}$ (red); $\Delta=3.2 \mathrm{kV}$ (black)). ${ }^{87} \mathrm{Rb}-$ ion detection efficiency (bottom) for a gain voltage of $2.7 \mathrm{kV}(\square)$. The acceleration voltage is the same as in (a).

In this Letter, we have shown a highly efficient, stateselective and fast ionization detection system for optically trapped neutral atoms which allows deterministic state analysis at the single-atom level. Though the detection process removes the analyzed atom from the trap, the system has a number of possible applications, for example combined with single atom traps it allows to determine partial and total photoionization cross sections free from ensemble averages [8] or together with coherent stimulated Raman adiabatic passage techniques the state analysis of Zeeman qubits [13]. Contrary to other approaches for singleatom state analysis, the geometry of the detection system provides a comparably large sensitive volume and high optical access. In the future it might thus be applied, e.g., for imaging [23] and site-specific readout of atoms in optical lattices where pulsed, scanning excitation schemes operating at high powers can enable readout times below $100 \mathrm{~ns}$ per atom. Furthermore, it could also be used for in situ, real-time probing of ultracold ensembles with subpoissonian accuracy [14] or as detector for a loophole-free test of Bell's inequality with a pair of trapped atoms at remote locations [13].

We acknowledge stimulating discussions with $T$. W. Hänsch, J. Volz, T. Schätz, H. Sjuts, and craftsmanship of the LMU workshop. Financial support was provided by the EU, the Elite Network of Bavaria, and the DFG through the projects Q-ESSENCE, QCCC, and MAP, respectively.

* florian.henkel@ physik.uni-muenchen.de

[1] Y. R. P. Sortais et al., Phys. Rev. A 75, 013406 (2007); M. K. Tey et al., Nature Physics 4, 924 (2008); R. Maiwald et al., Nature Physics 5, 551 (2009).

[2] I. Teper, Y.J. Lin, and V. Vuletic, Phys. Rev. Lett. 97, 023002 (2006); J. Bochmann et al., Phys. Rev. Lett. 104, 203601 (2010); R. Gehr et al., Phys. Rev. Lett. 104, 203602 (2010).

[3] R. Raussendorf, and H. J. Briegel, Phys. Rev. Lett. 86, 5188 (2001); O. Mandel et al., Nature 425, 937 (2003).

[4] M. L. Terraciano et al., Nature Physics 5, 480 (2009).

[5] A. Stibor et al. Phys. Rev. A 76, 033614 (2007); A. Günther et al. Phys. Rev. A 80, 011604(R) (2009).

[6] M. Weber et al., Phys. Rev. A 73, 043406-7 (2006).

[7] V. S. Letokhov, V. I. Mishin, and A. A. Puretzky, Prog. Quantum Electron. 5, 139 (1979).

[8] T. Dinneen et al., Opt. Lett. 17, 1706 (1992); C. Gabbanini, S. Gozzini, and A. Lucchesini, Opt. Comm. 141, 25 (1997); D. Ciampini et al., Phys. Rev. A 66, 043409 (2002).

[9] For later applied acceleration voltages of $3.8 \mathrm{kV}$, the Starkshifts of the Zeeman-sublevels of the $5^{2} S_{1 / 2}$ and $5^{2} P_{3 / 2}$ states are more than order of magnitude smaller than the natural linewidth of the transition. Thus, the spin-selectivity of the ionization process should not be affected.

[10] Type KBL10RS45-V2, Dr. Sjuts Optotechnik GmbH.

[11] The compensation electrodes modify the electric potential at the ioization region $(z=d / 2)$ such, that ions experience $71 \%$ of the applied acceleration voltage $U_{a c c}$. The remaining $29 \%$ of $U_{a c c}$ determine the kinetic energy of the electrons. With the current setup, the maximum applied acceleration voltage is $3.8 \mathrm{kV}$.

[12] If not specified otherwise the CEM gain voltage is $3.0 \mathrm{kV}$.

[13] J.Volz et al., Phys. Rev. Lett. 96, 030404 (2006); W. Rosenfeld et al., Adv. Sci. Lett. 2, 469 (2009).

[14] T. Campey et al., Phys. Rev. A 74, 043612-9 (2006).

[15] J. Fricke, A. Müller, and E. Salzborn, Nucl. Inst. Meth. 175, 379 (1980).

[16] M. P. Seah, and G. C. Smith, Rev. Sci. Instrum. 62, 62 (1991); I. S. Gilmore, and M. P. Seah, Appl. Sur. Sci. 144, 113 (1999).

[17] E.S. Fry, T. Walther, and S. Li, Phys. Rev. A 52, 4381 (1995); B. Brehm et al., Meas. Sci. Tech. 6, 953 (1995).

[18] J. V. Dunworth, Rev. Sci. Instrum. 11, 167 (1940).

[19] P. Kwiat et al., Appl. Opt. 33, 1844 (1994).

[20] C. N. Burrows, A. J. Lieber, and V. T. Zaviantseff, Rev. Sci. Instrum. 38, 1477 (1967); A. Egidi et al., Rev. Sci. Instrum. 40, 88 (1969); B. Tatry, J. M. Bosqued, and H. Reme, Nucl. Inst. Meth. 69, 254 (1969); J. Fox, R. L. Fitzwilson, and E. W. Thomas, J. Phys. E 3, 36 (1970).

[21] The statistical errors of the detection efficiencies [error bars in Fig[3 (b)] are given by the variances $\sigma_{\eta_{i, e}}^{2}=$ $\left(N_{c} / N_{e, i}^{\prime}\right)^{2}\left(1 / N_{c}-1 / N_{e, i}^{\prime}+2 N_{b e, b i} / N_{e, i}^{\prime 2}\right)$.

[22] The high gain voltages result from detector aging.

[23] K. D. Nelson, X. Li, and D. S. Weiss, Nature Physics 3, 556 (2007); T. Gericke et al., Nature Physics 4, 949 (2008); M. Karski et al., Phys. Rev. Lett. 102, 053001 (2009); W. S. Bakr et al., Nature 462, 74, (2009); J. Sherson et al., Nature 467, 68, (2010). 